\title{
Estenosis biliares benignas. Tratamiento endoscópico
}

\author{
María Elena López-Acosta* \\ Departamento de Gastroenterología y Unidad de Fisiología Digestiva, Hospital Ángeles Lomas, Huixquilucan Estado de México, México
}

\section{Resumen}

Se analiza la revisión de trabajos relevantes presentados durante ESGE Days 2019 y DDW 2019 en relación a las modalidades terapéuticas endoscópicas en estenosis biliares benignas, su eficacia y seguridad.

Palabras clave: Estenosis biliares benignas. Tratamiento endoscópico.

\section{Introducción}

La patología biliar está asociada a procesos inflamatorios como la colangitis esclerosante y otras colangiopatías autoinmunes, pancreatitis crónica (PC), coledocolitiasis $(\mathrm{CL})$ y cirugía, incluyendo derivaciones biliodigestivas, (DBD) colecistectomía (CLC) y trasplante hepático $(\mathrm{TH})$, e incluye entre otras complicaciones fugas biliares, estenosis (EB) y litiasis ${ }^{1}$. La colangiografía Endoscópica (CGE) es el procedimiento de elección en la mayor parte de estos pacientes, sin embargo otras opciones terapéuticas son necesarias en casos seleccionados como el drenaje percutáneo (DP), por ultrasonido endoscópico (USE) la cirugía y el retrasplante².

\section{Trasplante hepático (TH)}

Las complicaciones biliares se presentan en un 10 a 30\%, están asociadas a múltiples factores conocidos relacionados al paciente, las características del injerto, el tipo de cirugía: donador vivo (THDV) y donador cadavérico (THDC), criterios extendidos de donación y muerte por paro cardiaco (THDCMPC) y posoperatorio ${ }^{2}$. La dilatación endoscópica con balón y/o la colocación de endoprótesis plásticas (EP) ha sido un método estándar para tratar las estenosis biliares (EB) después del THDV ${ }^{3}$. En un estudio con seguimiento a cinco años, demostró que en el subgrupo de THCMPC, 34\% fueron diagnosticados con EB (20 anastomóticas (EA)14 no anastomóticas (ENA), realizándose 5,62 \pm 4 CPE por paciente, tratados con dilatación y colocación de EP, $2.87 \pm 1.3$, con una media 346 días respectivamente, con éxito inicial del $79 \%$, sin una diferencia significativa entre los pacientes con EA y ENA, ( $p=0,3)$, sin embargo, el éxito sin EP después de la CPE inicial se produjo antes en pacientes con EA y requirió menos procedimientos y más cortos $(P<0,01)^{4}$. En otro estudio se compararon 110 pacientes de 2005 a 2017 con drenaje unilateral (DU) vs bilateral (DB) en THDV del lóbulo derecho, pues lo general hay dos sitios de anastomosis incluyendo el conducto segmentario anterior derecho (CSAD) y el conducto segmentario posterior derecho (CSPD) el DU se realizó en 55 pacientes $(50 \%)$ y DB en 11 pacientes $(10 \%)$, sin diferencias significativas en la tasa de éxito clínico ( $80.0 \%$ vs. $90.9 \% ; p=0.669)$, ni tasa de complicaciones $(16.4 \%$ vs. $18.2 \% ; p>0.999)$ ni mortalidad a los 180 días ( $1.8 \%$ vs. $0 \%$; $p>0.999)$ pero durante el

\section{Correspondencia:}


seguimiento, 71 pacientes $(64,5 \%)$ requirieron DB más de una vez, mientras que solo 27 pacientes $(24,5 \%)$ alcanzaron la resolución con $\mathrm{DU}^{5}$. En un estudio retrospectivo, en 61 casos de TEDV, con EB, tratados con endoprótesis metálica autoexpandible completamente cubiertas (EMACC) por un año, se reportó éxito clínico de 67,2\% (41/61) y migración en el $54 \%$ (33/61), con tasa de recurrencia de $6,5 \%$ (4/61), la mayor distancia entre la estenosis y el hilio hepático se relacionó significativamente con el éxito del tratamiento (33.64 vs $28.31 \mathrm{~mm}, p=0.04$ ) y aunque no hubo otra diferencia estadísticamente significativa, el diámetro del conducto hepático común más delgado del donante se relacionó con migración (7.97 vs $9.38 \mathrm{~mm}, \mathrm{p}=0.06$ ) y el diámetro del conducto hepático común del donante más grande con recurrencia de la estenosis (11.20 vs $8.4 \mathrm{~mm}$, $p=0.06)^{6}$. En una revisión sistemática y un meta-análisis para evaluar la eficacia y la seguridad de las EMACC vs las EP, que incluyó cinco estudios, con 221 casos, tres no mostraron diferencia estadísticamente significativa, relacionada a la resolución de la EB (Riesgo relativo (RR) 0,96 [IC 95\% 0,83 a $1,11, p=0,62, I 2=43 \%, p=0,17)$ y a la recurrencia (RR 1,51 (IC 95\%: 0,36 a 6,26), $p=0,57, \mathrm{I} 2=60 \%$, $p=0,08)$. En los cinco no hubo diferencias en complicaciones (RR 1.30 [IC del 95\%: 0.59 a 2.83], $p=0.52, \mathrm{I} 2=41 \%, p=0,15$, ni en mortalidad por otras causas (RR 1.46 [IC del 95\%: 0.25 a 8.60], $p=0.68,12=0 \%, p=0.72$ ) y el éxito del tratamiento (RR 1.04 [95\% IC 0,93 a 1,17], $p=0,47$, I2 = no aplicable). La evidencia de certeza moderada sugiere que no existe diferencia entre la eficacia y la seguridad EMACC vs EP en $\mathrm{TH}$, en EB, de baja certeza también sugieren una similitud de los efectos de ambas prótesis estudiadas sobre la recurrencia de las estenosis biliares y sus complicaciones?.

\section{Estenosis biliares posquirúrgicas}

Las EB pueden ser refractarias a la terapia endoscópica y no hay consenso sobre el período óptimo de permanencia de la endoprótesis y sus recambios, se presentó un trabajo sobre la utilidad de EP interna vs convencional, para 16 pacientes la duración media de la permeabilidad para el EB interno y externo fue de 222 (rango; 8-1736) días y 99 días (rango; 7-356), respectivamente sin diferencias en complicaciones ${ }^{8}$, así mismo en EB proximales se presentó un trabajo con una EMAE completamente cubierta, modificada, corta, con recambios cada 3 meses, en 106 pacientes a tasa de éxito técnico fue del $100 \%$ y la tasa de éxito clínico fue del $95,3 \%$. La tasa de recurrencia fue del $19,8 \%$ y la tasa de complicaciones fue del $8,5 \%$. Todas las prótesis fueron removibles y la migración se observó en 3 pacientes $(2,8 \%)^{9}$. Un estudio retrospectivo que incluyó 200 pacientes tratados con una EMACC anclada vs no anclada, se encontró seguro y efectivo, con menos intervenciones ${ }^{10}$. Se presentó la experiencia inicial con nuevas endoprótesis biodegradables (EBD), están fabricadas con una mezcla de polímeros que se biodegradan en diferentes intervalos: lento (11 semanas), medio (20 días) y rápido (12 días), en estenosis benignas biliares y pancreáticas, evaluando tiempo de biodegradación, mediante placas de abdomen (2/4 semanas rápido, 3/6 semanas medio y 3/6 meses lento), y los efectos adversos, en comparación con EP. Fueron 22 pacientes (32 EB). En estenosis pancreáticas se demostró degradación parcial a las 2 semanas y completa a las 4 , en $4 / 5$ casos con migración en uno, en los 6 restantes (2 medio y 4 con lento) mostraron degradación completa del EB en el tiempo esperado, lo anterior sugiere que la colocación de EBD es factible, con adecuada visualización mediante fluoroscopia y perfil de seguridad favorable ${ }^{11}$. En estenosis refractarias, se ha descrito las inyecciones de esteroides y la incisión con aguja y cuchillo, y una nueva endoprótesis metálica intraluminal por aposición (lumen apossing metal stent LAMS), se presentó un metaanálisis que incluyó 34 estudios (LAMS: 8; EMACC: 15; EBD: 11), concluyendo que las LAMS parecen tener un mejor perfil de seguridad. La migración fue más notoria con EMACC y la hiperplasia tisular fue más notoria con EBD. El dolor postoperatorio fue comparable en EMACC versus EBD y significativamente más bajo con LAMS. La comparación indirecta, los sitios de EB variable y la heterogeneidad fueron las limitaciones de este análisis ${ }^{12}$. La incidencia de estenosis refractarias se ha descrito entre el 10 y el $22 \%$. Se describió la utilidad de la dilatación combinada con la inyección de esteroides guiada por colangioscopia en tres pacientes post TH ortotópico, con seguimiento medio fue de 26 meses, con recaída y reconstrucción quirúrgica en uno y éxito en dos, las limitaciones de este estudio son el tamaño de la muestra y su experiencia como centro único, pero es una opción atractiva ${ }^{13}$. Para estos casos se reportó la experiencia en el tratamiento endoscópico de 4 pacientes con estenosis recidivantes biliar y pancreáticas, en pancreatitis crónica y $\mathrm{TH}$ con el uso de la punta de cistograstrostomo guiado (Cystogastrostoset, Endoflex, bajo control endoscópico, usando colangioscopia, SpyGlass, con aplicación de corriente de 
corte hasta lograr franquear la estenosis y colocar EP, con drenaje adecuado, de manera segura, con éxito clínico en todos los casos ${ }^{14}$.

\section{Complicaciones}

Los factores de riesgo de lesión duodenal debida a la migración de EP siguen siendo inciertos, se revisaron de manera retrospectiva doscientos cuarenta y ocho pacientes con 402 procedimientos de CGE. La incidencia de migración distal fue del 6,2\%. La frecuencia de lesión duodenal fue del $2,2 \%$ en todos los casos y del $36 \%$ en los casos con migración distal. Las EB, la longitud del EP sobre el extremo proximal de la estenosis $(\geq 2 \mathrm{~cm})$ y la duración de la retención ( $<3$ meses) se asociaron de forma independiente con la migración distal ( $p=0.018, p=0.009$, y $p=0,016$, respectivamente). La lesión duodenal ocurrió con más frecuencia en casos con un ángulo mayor $\left(\geq 30^{\circ}\right.$ ) entre el extremo distal del EP y la línea central del cuerpo del paciente $(p=0.018)$ $o$ en casos con retención de $<3$ meses $(p=0.031)^{15}$.

\section{Conclusiones}

La CGE es la opción terapéutica más efectiva y segura en el tratamiento de las EB benignas, con dilatación y colocación de EP o EMACC, con diferencia en el porcentaje de complicaciones como migración y número de procedimientos, las nuevas EBD, EMACC y LAMS ofrecen nuevas opciones en efectividad y en caso de refractariedad se cuenta con otras opciones como inyección de esteroides y aplicación de corriente mediante colangioscopia, antes de considerar otras opciones como el drenaje percutáneo, USE y la reconstrucción mediante cirugía.

\section{Conflicto de intereses}

No existen conflictos de interés. Sin patrocinio de la industria.

\section{Bibliografía}

1. The role of ERCP in benign diseases of the biliary tract Clinical Guideline. Gastrointestinal Endoscopy Volume 81, No. 4: 795-803. 2014.

2. Stenting for benign and malignant biliary strictures. Jason B. Mullady D. Gastrointestinal Endoscopy Clinics of North America. Volume 29 Issue 2. 215-235.2019.

3. Endoscopic Management of Biliary Issues in the Liver Transplant Patient. Crismale, J F, Jamad A. Gastrointestinal Endoscopy Clinics of North America Volume 29 Issue 2. 237-256.2019.

4. Outcomes of endoscopic therapy in donation after cardiac death liver transplant biliary strictures Divyanshoo R. Kohli et al. Abstract. Poster Session. DDW 2019.

5. Unilateral versus bilateral biliary drainage in patients with biliary anastomotic strictures after right-lobe living donor liver transplantation. Min Su You et al. Abstract. Poster Session. DDW 2019.

6. Can we predict endoscopic treatment outcome of biliary anastomotic stricture after orthotopic liver transplant? Fernanda P. et al. Abstract ESGE Days Praga 2019.

7. Covered self-expandable metallic stent versus plastic stents for anastomotic biliary strictures after liver transplantation: a systematic review of randomized controlled trials. Moribe D. et al Oral Session DDW San Diego 2019.

8. Endoscopic stent placement above the sphincter of oddi for biliary strictures after living donor liver transplantation. Koizumi $\mathrm{M}$ et al. Poster Session DDW San Diego 2019.

9. A fully covered self-expandable metal stent with special design is useful in patients with high level biliary stricture after biliary operation Sung Jang et al. Oral Session DDW San Diego 2019.

10. A comparative analysis between anchoring and not anchoring fully covered self-expandable metal stents in the management of benign and malignant biliary strictures Shawn et al. Poster Session DDW San Diego 2019.

11. Feasibility of new biliary and pancreatic biodegradable stent placement: interim analysis of an ongoing single-center, prospective pilot study. Anderloni Andrea et al. Poster Session DDW San Diego 2019.

12. Efficacy of lumen-apposing metal stent in the treatment of refractory benign gastrointestinal strictures in comparison to fully-covered self-expanding metal stent and biodegradable stent: a systematic review and meta-analysis, Babu P. et al. Oral Session DDW San Diego 2019.

13. Cholangioscopy guided steroid injection for refractory post liver transplant anastomotic strictures: an alternative rescue approach. Mitsuhito K. et al. Poster Session DDW San Diego 2019.

14. Endoscopic management of difficult bening biliary and pancreatic strictures using a wire-guided cystotome.Magas San-Juan Carolina, et al Poster Session. ESGE Days 2019.

15. Risk factors for distal migration of biliary plastic stents and related duodenal injury Yuan X. et al. Poster Session. DDW San Diego 2019. 